\title{
Providing care quality by design: a new measure to assess hospital ward layouts
}

Rosica Pachilova

Space Syntax Laboratory, The Bartlett School of Architecture, University College London, London, UK, rosica.pachilova.10@ucl.ac.uk (corresponding author)

Dr Kerstin Sailer

Space Syntax Laboratory, The Bartlett School of Architecture, University College London, London, UK, k.sailer@ucl.ac.uk

Published in the Journal of Architecture, Volume 25, 2020, issue 2, p.186-202 


\title{
Providing care quality by design: a new measure to assess hospital ward layouts
}

\begin{abstract}
Which hospital ward layout works best? In the past, one response to this question has been to design layouts that minimise walking distances of healthcare workers, and increase the time spent with their patients. However, new research suggests that good face-to-face communication between doctors and nurses crucially impacts the health and safety of patients. Taking this into account, this research proposes a new single measure called Spaces for Communication Index (SCI). It assesses communication opportunities arising from the layout, and shows that a high index is associated with the provision of good healthcare. Six NHS wards were first studied in depth by collecting detailed information about movement and communication patterns of healthcare workers. The index was developed on this basis. Thirty-one NHS wards were then selected, based on their quality of care rating. These were used to test the index. Each ward was analysed with the Space Syntax method, which investigated the size of visual fields of healthcare workers on everyday movement paths through the ward. Large viewsheds provide good visibility and awareness of the environment. As a result, they accrue more communication opportunities by virtue of the layout. Statistical analysis was also used to test if the index can predict care quality. Other factors such as distances between key areas, number of patient beds or ward size were tested, too. Results showed that the higher the index, the better the quality of care. The other factors were not related to healthcare quality. In terms of design, these results high- light the importance of the openness of spaces that healthcare workers traverse to get from one key area to another. This research contributes to the development of an objective method that designers can use to compare different nursing unit designs, and anticipate the care quality that would be provided to patients.
\end{abstract}

\section{Introduction}

Hospital planners and architects who undertake the difficult task of designing a hospital ward have to choose from a variety of types of ward layouts. These include radial, racetrack, and straight or L-shaped corridor layouts (Fig. 1). How to best design a ward when there are so many different typologies on offer? To assess which layout 


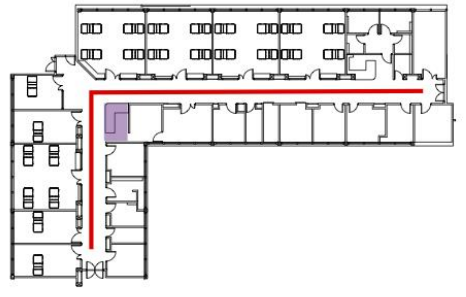

Radial layout

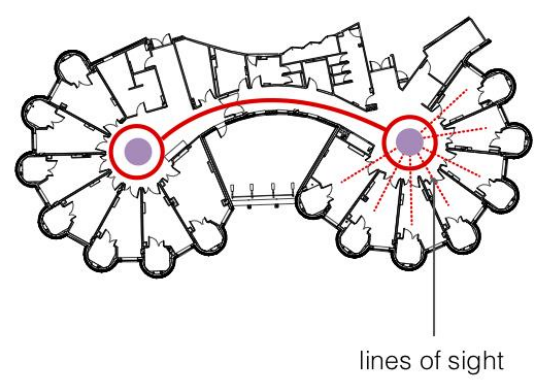

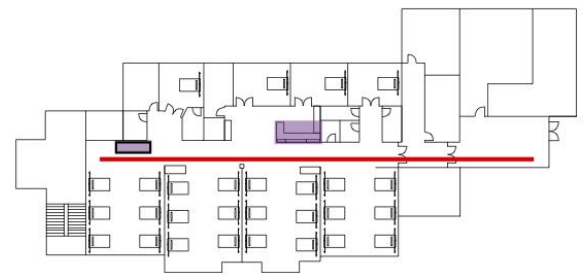

Racetrack (double corridor) layout

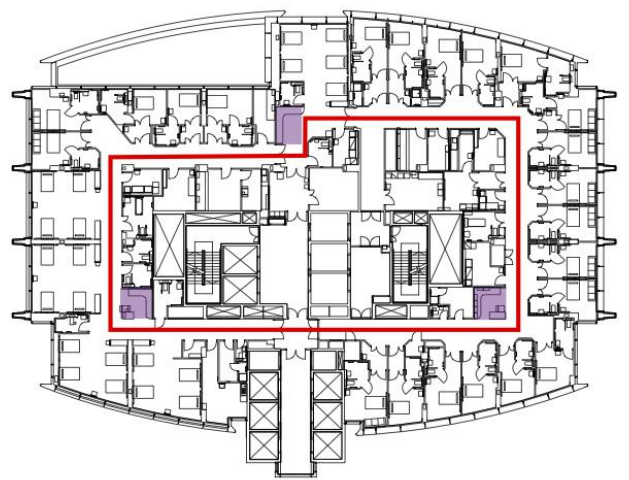

Figure 1. Hospital ward typologies. A red bold line indicates the main corridor, while the nursing station is in purple. The dark purple lines in the 'triplex layout' denote each patient bay with its own central nursing station

performs best, previous research has suggested that minimising walking distances between functional areas of a ward (e.g. patient beds and nursing stations) increases the time healthcare workers spend at the patients' bedsides. ${ }^{1}$ It is therefore possible to infer which areas of the inpatient ward should be placed in close proximity. Such measurements have been applied to different ward typologies to define the most efficient one, with the assumption that this would also be the best performing one. However, studies showed disparate results, each favouring a different ward typology over another. ${ }^{2}$

Beyond walking efficiency, prior research has also highlighted another factor. These studies suggest that the spatial configuration of hospital wards influences face-to-face communication among healthcare workers.

Communication is of high importance in healthcare environments, as it is also crucial for the health and safety of patients. A study of an inpatient ward showed that verbal miscommunication between nurses and doctors was responsible for $37 \%$ of all errors. ${ }^{3}$ In another study of 5,030 patients in twelve nursing units, William A. Knaus and his colleagues found that collaboration among healthcare workers was the strongest factor contributing to observed differences in patient outcomes. ${ }^{4}$ These researchers pointed to the importance of the visibility of the layout. This provides opportunities for interaction between healthcare workers, especially as it supports informal and unplanned conversations that take place on the go, and thus minimise communication errors. These studies investigated the relationship between the spatial properties of a layout and communication as a proxy for healthcare quality. But they did not link the spatial design of a ward to care quality. 
Thus, existing research on ward typology and spatial configuration failed to describe the 'best' layout. This research addresses the ways in which the layout of hospital wards influences not only walking practices or distances, but also outcome measures such as care quality. The research aimed to develop a new single measure, by means of which design schemes for nursing unit layouts can be benchmarked for healthcare quality. The measure was called Spaces for Communication Index (SCl). Rather than measuring space efficiency in terms of minimising distances, this index measures space effectiveness in terms of maximising opportunities for communication. These are crucial for providing good healthcare. This new index $(\mathrm{SCl})$ aims to help a designer assess ward designs, and anticipate which spatial configuration may contribute to healthcare quality.

The first section discusses research studies that investigated the effect of ward typologies on nursing walking patterns. It also reviews studies that focus on the importance of spatial configuration for communication and exchange of information. The second section describes the case studies and methods used for the analysis. The third section discusses the basis for calculating SCI. It also explains how the index was further developed using results from statistical analysis of thirty-one case studies, and correlations with healthcare quality metrics. The final section discusses the significance of $\mathrm{SCl}$ in hospital ward design, and summarises the conclusions and limitations of the study.

\section{In search of the ultimate hospital ward layout design}

\section{Comparing hospital wards based on ward typology}

Several studies reported that dense radial layouts (Fig. 1) can be associated with shorter walking distances for the nurses, improved patient monitoring, and better work efficiency. In 1960, Madelyne Sturdavant compared two intensive care units, one with a radial and one with a single-corridor design (Fig. 1). ${ }^{5}$ She found that in the radial layout, nurses walked less frequently to patient rooms because this design enabled increased visual supervision of the patient from the nursing station. In addition, the average time spent within patient rooms was equivalent to that in the single-corridor layout. Increased patient visibility in the radial unit also enabled increased participation of nurse practitioners and managers in patient care than the single-corridor unit. The study favoured radial layouts, but the typology was compared only with one alternative ward type.

David Trites and colleagues compared the behavioural and perceptual influence of three different unit designs in the same hospital: four radial units, four double-corridor units, and four single-corridor units (Fig. 1). ${ }^{6}$ A total of 590 staff members were observed over a period of eighty-two days, and data on time utilisation of the nursing staff was also collected. In terms of efficiency, the radial design was deemed more successful because nurses travelled significantly less ( 0.61 miles on a day shift) compared to the other two designs ( 0.79 miles on double-corridor units, and 1.08 miles on single-corridor units). After interviewing staff members who had been transferred between a radial and a double-corridor unit, the researchers also reported that differences in layout could influence the way nursing staff members work together. More 
specifically, staff members transferred to the double-corridor unit reported that teamwork was poorer, and those transferred to the radial unit reported that teamwork was more effective after the transfer. More significantly, interviews and questionnaires indicated that $72 \%$ of the patients involved in the transfer preferred the radial unit over the double-corridor unit. The primary reason given for this preference was the patients' feeling of better observation and faster response by nurses in the radial design. These studies compared a larger number of units. They did not only look into travel distances, but they also discussed the importance of layout visibility for teamwork and patient satisfaction in different ward typologies. Results confirmed their findings from the previous study. In conclusion, the radial layout was not only more efficient, but also more effective for teamwork. It was also preferred by patients.

Another set of studies, however, reported that the double-corridor layout was the most efficient in minimising travel distances. In 1967, John Freeman developed a model that combined frequencies of nursing traffic between functional points on the unit with distances and area measurements, appropriate wage rates, and construction cost indexes. ${ }^{7}$ The combined costs for the unit were used as a measure of efficiency. In 1968, Richard Sendler applied the model developed by Freeman on fourteen nursing wards of various shapes, numbers of patient beds, and arrangements of functional points. ${ }^{8}$ The author concluded that layout was more important than size in determining the efficiency of inpatient nursing units. The double-corridor layout was the most efficient, followed by the radial and single-corridor layouts. He also found that nursing units with compound circulation were more efficient than those with simple circulation. Since this measure included travel distances and costs of amortisation of construction, these results favoured a different ward typology.

In 1975, John D. Thompson and Grace Goldin used link analysis to develop the Yale Traffic Index. This was used to compare general medical and nursing units for functional efficiency. ${ }^{9}$ Thompson and Goldin identified sixteen areas on a typical nursing ward, and recorded the number of trips between each pair of areas, which were referred to as 'links'. They found that more than $91 \%$ of the traffic on the unit could be accounted for by fourteen links that involving seven out of the sixteen areas. These links were considered to be the prime determinants of unit efficiency. The relative trip frequencies of these fourteen links were multiplied by distance measures, and their result comprised the 'Yale Traffic Index'. This index was then used to comparatively evaluate units with similar facilities but different layouts. The smaller the index, the shorter the travel distance, and thus the more efficient the layout. The method was applied to thirty inpatient units. Thompson and Goldin found that wards with a redundant circulation scheme (i.e. double-corridor units or circular plans) were more efficient than the simple ones. They also concluded that inpatient ward efficiency is not directly related to its size or degree of privacy.

In general, however, no single geometry was found to be superior in all respects. ${ }^{10}$ Studies of unit typologies eventually shifted to debates on the distribution of nursing stations, and comparisons between different types (such as centralised versus decentralised stations). While the use of these overall typologies can simplify high-level planning, overgeneralisation and oversimplification of the layouts to different unit types or different nurse station types failed to capture fundamental differences that influenced work patterns. 


\section{Comparing hospital wards in terms of spatial configuration}

The same period witnessed the emergence of Space Syntax. Developed in University College London, this new theory and method of analysis aimed to describe spatial layouts in a more fine-grained manner. ${ }^{11}$ Space Syntax investigates how spatial elements such as the rooms and corridors of a building are interconnected in a spatial network. It also studies how the resulting system influences people's visibility and movement, and therefore their behaviour, and communication.

Space Syntax quantifies spatial elements based on their relationship with other elements in the spatial system. It is a method of representing our perception of space, and its potential use. It assesses the complexity of design layouts in terms of the number of connections and distances between spaces. It shows how well-connected or isolated, and therefore integrated or segregated, a space is within a building or urban complex. Space Syntax offers researchers and designers a sophisticated method to comparatively analyse different layouts (in our case, hospital wards) of the same typology. A growing body of research in the field has shown that there is a relationship between the spatial design of buildings, and the ways in which people socially engage with them. ${ }^{12}$

Previous research studies in hospital environments showed that the spatial properties of a layout influenced healthcare workers' movement and interaction patterns. In 2010, Ruchi Choudhary and her colleagues used space syntax measures to develop an empirically derived statistical model to predict the effect of different hospital ward layouts on nurses' movement patterns. ${ }^{13}$ They developed this tool because there were no simulation models that could successfully distinguish between two layouts that had roughly similar path distances and number of facilities but were fundamentally different in their design or spatial organisation. The researchers used a data set of fifty-three nursing activities in five different hospital floors. In common with the Yale Traffic Index, they identified major movement paths from several work areas, including patient beds, nursing stations, and support areas. They aimed to model the nurses' random and unplanned movement, which was affected by the spatial and visual properties of the environment, and was not accounted for by simulations. The authors argued that nurses usually worked and moved within their assigned sub-area of the unit. They were more likely to modify and adapt their behaviour to the characteristics of their sub-area rather than the design of the entire medical unit. Hence, they focused only on the sub-area a nurse would move around in, since this could be substantially different from the overall unit layout. Results showed that the number of trips to the patient rooms was strongly influenced by the spatial properties of the sub-area. Subareas with larger viewsheds and shorter path length resulted in fewer trips to patient rooms because visibility was better. This study highlighted the importance of visibility for healthcare workers' movement and interaction patterns. The researchers developed a rigorous model that could potentially be used in the design of nursing units. But before it could become applicable, further work was required. This model was used to inform the development of SCl.

In another study where the visual properties of a layout were investigated in detail, Yi Lu and her colleagues investigated how visibility affected behavioural activities and communication of doctors and nurses in an intensive 
care unit. ${ }^{14}$ In addition to generic visibility, the authors developed a new measure: targeted visibility analysis. While generic visibility was calculated from all visible points to all other points in space, targeted visibility was calculated towards a number of pre-selected targets (in this case, patient beds). The researchers found that targeted visibility was more strongly correlated with the location of nurses, and the location of interacting nurses. This meant that more nurses could be expected in areas with higher visibility (in other words, with larger viewsheds towards patient beds). Generic visibility was more strongly correlated with the location of doctors. This could be explained by their preference for a position that would maximise their awareness of the surrounding environment.

In a later study, Lu and her colleagues applied the targeted visibility measure to a data set, collected for a different study by David E. Leaf and his colleagues, to put the new tool into further empirical testing. ${ }^{15}$ This time, the data included patient outcome variables (such as hospital mortality rate, ICU mortality rate, and average length of stay for patients). These allowed the authors to relate their new spatial measure to a healthcare outcome. They chose to work with this specific data set because Leaf and his colleagues were the first to draw any association between patient mortality and room visibility. But their definition of visibility was rather vague. Leaf and his colleagues collected data from twelve rooms in a medical intensive care unit including information about 664 patients, and Lu and her colleagues calculated various visibility measures (such as patient head visibility, patient room visibility, and field of view to the rest of the unit), using targeted visibility. Their results showed that the most acute patients had significantly higher ICU mortality rates when assigned to a low-visibility room. The mortality rates of less serious cases of patients were not affected. Lu and her colleagues developed a promising tool that accounted for the visibility relation between the nursing station and patient rooms in an intensive care unit. But this measure was only tested in one case study.

In 2014, Mahbub Rashid and his colleagues conducted an empirical investigation in four adult intensive care units. They studied how the visibility levels of a layout could be used to explain interaction behaviours of healthcare workers. ${ }^{16}$ Units of different size, geometry, and speciality were selected to be studied in detail. Results showed that when nurses and doctors interacted while sitting, they preferred spaces with good global visibility to maintain high levels of environmental awareness. But when they communicated while walking, they preferred spaces with large viewsheds that gave them higher control over neighbouring spaces. The authors argued that such consistent behavioural patterns occurred due to the structural similarities of the spatial con-figuration of the four wards. It was these 'spatial genotypes' ${ }^{\prime 17}$ that played the crucial role here, over and above the general functional similarities of intensive care units, and their different sizes and geometries. This research showed that size and geometry may be irrelevant for communication. What matters is the configuration of the ward.

A few important conclusions can be derived from the above studies. First, spatial configuration, and especially visibility in hospital wards, matters for movement and interaction. However, the results of existing studies are not always consistent. In the study by Lu and her colleagues, doctors and nurses 
were tuned to different visibility settings. But Rashid and his colleagues found similarities between the two groups despite differences in behaviours. ${ }^{18}$

Second, researchers in the field attempted to develop rigorous tools and models that measure the visual properties of a layout and predict the effect on care providers' movement and interaction patterns. But these studies either required further work in the development stage of the new measures, or did not test their tools and models on a sufficient number of case studies. Third, the configuration of the nursing unit assignment (sub-area) may be more important than the whole unit layout. Finally, despite functional differences of nursing unit layouts, it is still possible to identify structural spatial similarities, and use them for the development of a universal measure. Our aim was to develop an index applicable to all types of inpatient wards. We therefore used the logic behind the Yale Traffic Index, the model by Choudhary and her col- leagues, and the nursing unit assignment to develop SCl.

The next section will discuss the case studies we selected for the development and testing phases of SCl.

\section{Case Studies}

In a first stage, three UK National Health Service (NHS) hospitals were selected as case studies to develop the tool. Six inpatient wards (one intensive care unit in each hospital, one medical/surgical ward in two of them, and one labour ward in the third) were selected to be analysed. We deliberately selected units of different spatial layouts and sizes. Our sample included two wards with a race- track (double-corridor) layout, three with a single-corridor layout, and one with a triplex layout (where three bays with a central nursing station each shared support facilities) (Fig. 1). Ward sizes ranged from $470 \mathrm{~m}^{2}$ to $1500 \mathrm{~m}^{2}$, or from nine to forty-three patient beds. Floor plans of the hospitals were obtained either from the hospital architects or the hospital management teams.

In a second stage, thirty-one NHS hospital wards from eleven hospitals were selected to further test the tool. Hospitals were selected based on their quality of care ratings. Floor plans were obtained through the Freedom of Information Act, which allows members of the public to request information from the related public authorities. A representative sample of hospitals with different ratings was selected to be analysed. The ratings deemed five of these wards 'outstanding', eleven 'good', two 'inadequate', while the other thirteen 'require[d] improvement'. For the purposes of statistical analysis, the last two categories were merged into one. Ward sizes ranged from $382 \mathrm{~m}^{2}$ to $2300 \mathrm{~m}^{2}$, or from nine to fifty-two patient beds. Wards also varied in terms of the number of single patient rooms. Some examples had $100 \%$ patient rooms, while others were $100 \%$ open bays. All examples had one or several centralised nursing stations, but none of them had only decentralised nursing stations. The wards varied in their typology with examples from racetrack, single straight corridor, single Lshaped corridor, radial and triplex layouts (Fig. 1). The wards differed in their specialty too. Examples included intensive care units, medical/surgical wards and labour wards. 


\section{Methods}

\section{Space Syntax}

The method of Space Syntax was employed to analyse each of the initial six case studies and the following thirty-one wards. Visibility graphs were used to rep- resent the spatial configuration. These graphs are spatial representations based on isovists. ${ }^{19} \mathrm{An}$ isovist illustrates the visible areas around a generating point in space in 360 degrees (Fig. 2). A visibility graph works by overlaying a human-scale grid spacing of $60 \times 60 \mathrm{~cm}$ on top of a floor plan, constructing an isovist from the centre of each pixel of the grid, and connecting every pixel to the pixels that can be seen within the isovist area (Fig. 3). Based on these connections, different measures can be calculated. These assign values to every pixel. This representation provides a description of space 'from inside'. It describes space from the point of view of individuals, as they perceive, interact. and move through it.

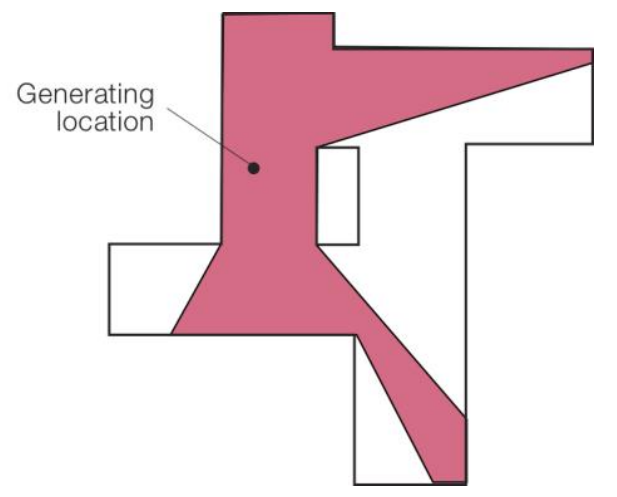

Figure 2. An isovist polygon incorporating the visible area from a generating location.
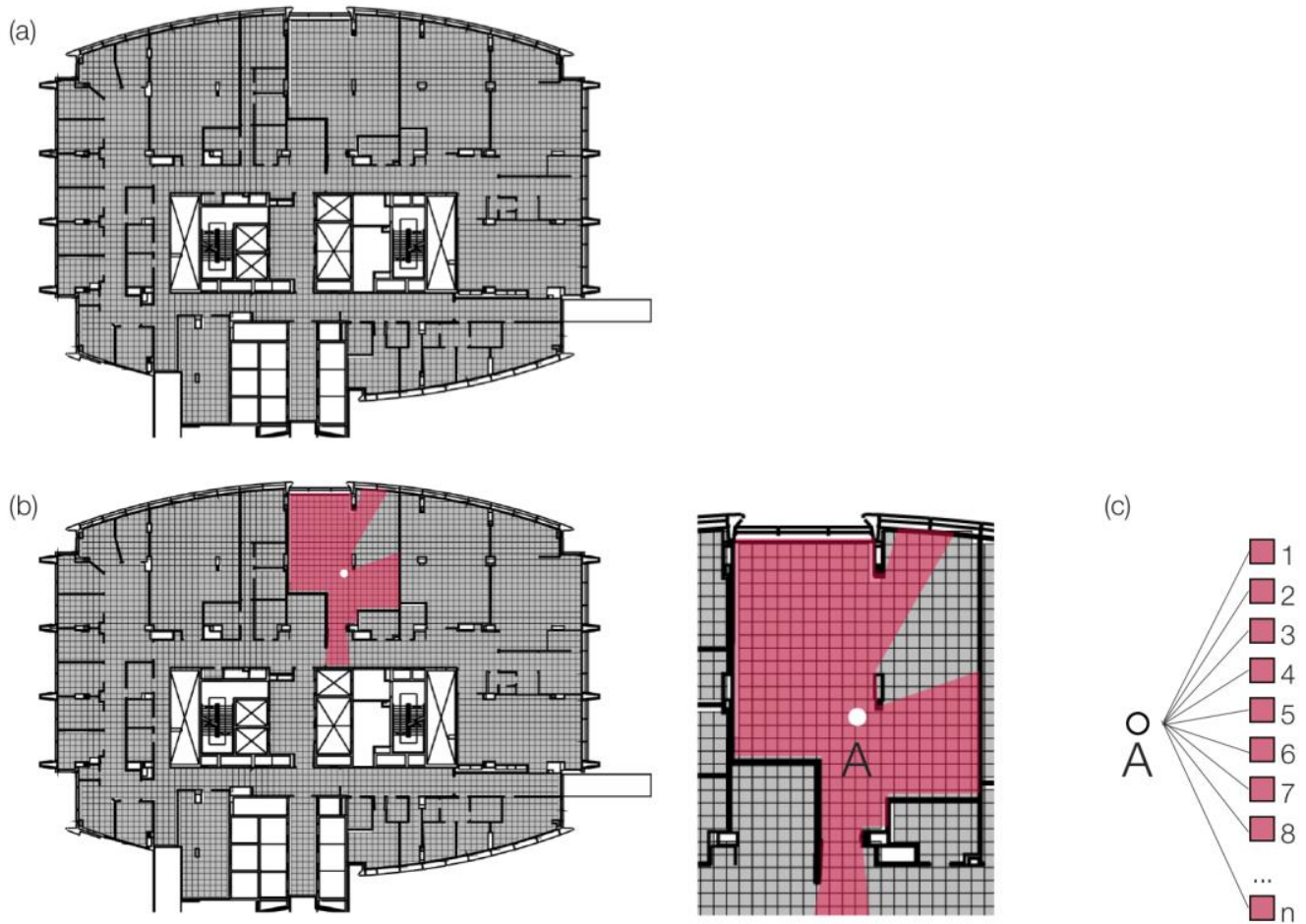

Figure 3. (a) A grid spacing $60 \times 60 \mathrm{~cm}$ overlaid on top of a floor plan to represent space from a human perspective; (b) visible pixels based on an isovist from a selected pixel; (c) construction of a graph of pixels (nodes) and links (ties) 
Depthmap X software ${ }^{20}$ was used to generate and analyse the visibility graphs. Visibility Graph Analysis (VGA) was constructed using only solid walls modelled as barriers thus representing what one can see in space from a standing position. ${ }^{21} \mathrm{~A}$ red-to-blue colour scheme was used to visualise how connected a space within a ward was. Highly connected areas were shown in warm colours and highly segregated areas in cool colours (Fig. 4).

To study visibility relationships, the local measure of connectivity was used. Connectivity measures the number of spaces immediately connected to a space of origin (how many pixels are in the immediate visual field of a pixel of origin; in other words, how many pixels fall in the isovist of a pixel). Hence, connectivity denotes the size of a viewshed from a specific vantage point. We worked with connectivity because previous research showed that interactions between caregivers in a hospital were influenced by the connectivity levels of spaces. ${ }^{22}$
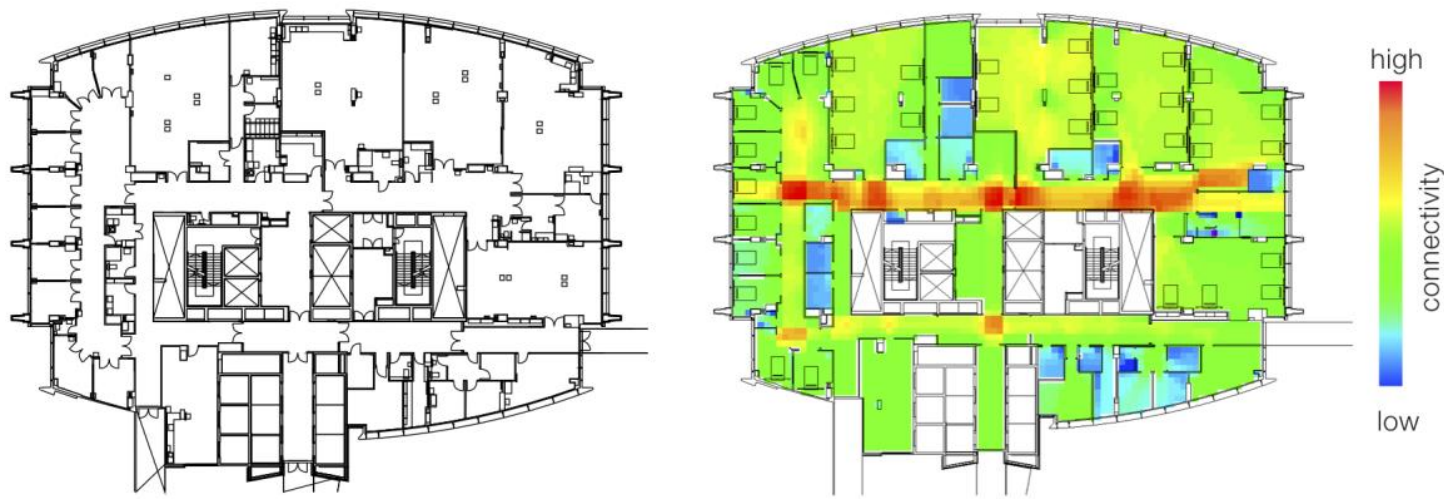

Figure 4. (left) A floor plan used to model a visibility graph on eye-level using only solid walls as barriers; (right) visualisation of the measure of connectivity, showing highly connected spaces as warm colours and spaces with low connectivity as cool colours

\section{Direct observations of movement and communication patterns}

To understand movement patterns and communication, direct observations of care processes were carried out in the six initial wards, using a method called 'people shadowing'. Across all six cases, a total number of 102 healthcare workers (including medical, nursing, and ancillary staff) were followed by an observer for an average of forty minutes each while they went about their work. Observations were conducted during day shifts between 8:00 am and 8:00 pm over the course of several days. The total duration of observations was seventyfive hours and thirty-four minutes. A pre-programmed Excel spreadsheet on a tablet was used to record every space the observed participant occupied, and the type and duration of activities they performed there. A number of different activities were observed, but this analysis focused especially on walking, and face-to-face conversation. The collected data provided information on frequency of space usage (e.g. how many times a healthcare worker went to the nursing station), movement paths, and location and frequency of conversations. This information was used in the development phase of SCl to inform the selection of paths from/to key areas. To conduct these observations, ethical approval was granted by the UCL Research Ethics Committee. 


\section{Quality of Care}

Ratings from the Care Quality Commission (CQC) were used to rate the thirty-one hospital wards on their healthcare quality during the testing phase of $\mathrm{SCl}$. CQC is an independent regulator of health and social care services in the United Kingdom. It conducts inspections of hospitals and rates them on a fourpoint scale, ranging from 'outstanding' through 'good' to 'requires improvement' and 'inadequate'. This rating is based on a number of data sources including evidence and information collected from observations of daily work activities, interviews with staff and patients, and inspections carried out by experts. We specifically selected this care quality rating because the factors they monitor are diverse. These include patient safety and care, team effectiveness, dignity and respect of patients, responsiveness to patient needs, and leadership and management of the hospital. The data is also freely available on the CQC website, which provides healthcare quality information for specific services (such as intensive care units), and overall, on the hospital level.

In addition to these measures, numbers of patient beds, ward sizes, and the Yale Traffic Index were also calculated, and used for the analysis.

\section{Analysis and results}

This section will first discuss the basis for calculating SCI, followed by a discussion of the development and testing phases. The Space Syntax method dis- cussed in the previous section was used in all stages of the process. The data from direct observations of activities was used in the first two stages. The quality of care rating was used in the final testing phase.

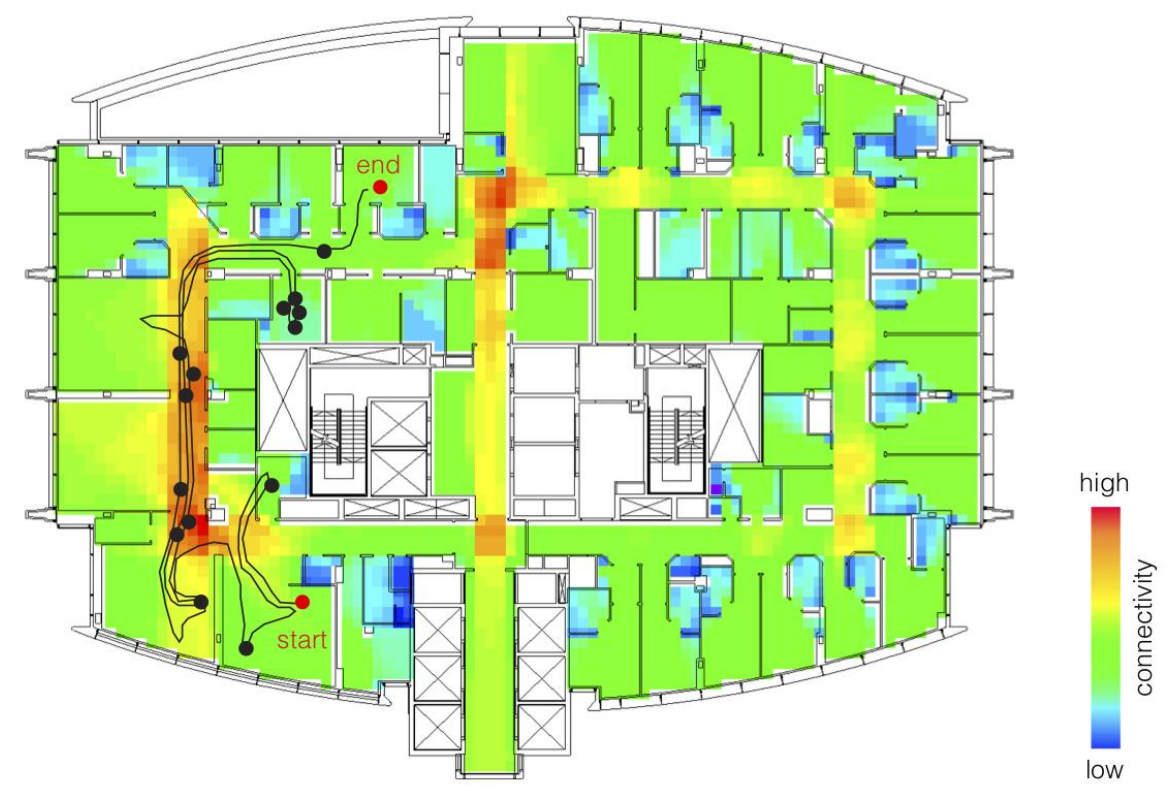

Figure 5. A diagram illustrating how accumulated connectivity of a healthcare provider was calculated in one of the hospital wards where the black dots represent the location of conversations, the black line - the walking path of a caregiver, and the red dots - the start and end-points of the path 


\section{Phase 1: SCl basis - accumulated connectivity; or, how much exposure one has to meet other people}

Since people are not static, and even more so in healthcare environments, doctors and nurses have quite a dynamic movement pattern. They can be going back and forth from a space with high visibility to a space with low visibility. Along the way, they 'accumulate' levels of connectivity. Our study was based on this main hypothesis: The higher the accumulated connectivity along the work path of a healthcare worker, the more opportunities for inter- action they have been provided with. To test this hypothesis, the walking paths of every observed participant in the six case studies were drawn on top of the floor plan (Fig. 5). We calculated how much connectivity they accumulated along their paths by multiplying the connectivity of each area they passed through by the time they spent there. For example, if the connectivity of a nursing station was 230 , and a healthcare worker stayed there for twentythree seconds, the accumulated connectivity was calculated by multiplying 230 $\times 23$. Since this was done for every space along their path, the total accumulated connectivity by healthcare worker was calculated by summing up all numbers accumulated along the way. Thus, every healthcare worker had a score for accumulated connectivity. The total number of conversations they had along their way was also calculated. We were especially interested to test whether more conversations would take place, if more connectivity was accumulated during a shift.

The statistical model selected for this data analysis, as it was more appropriate for the structure of the dataset, was a negative binomial regression. This was used to test the relationship between communication and accumulated connectivity. Results showed that the higher the accumulated connectivity along the work paths of care providers, the higher the chances of communicating with others. These results were statistically significant. This means that they cannot be attributed to chance. The probability value (also known as p-value) was less than 0.0005 . This means that the probability of getting a different result was less than 0.5 times in 1000. Accumulated connectivity can therefore be considered as a measure that indicates how much exposure one has to meet other people. It can be used as a predictor of the number of communication instances a healthcare worker would have.

'This is great!' a healthcare planner or designer might exclaim. 'But how can I apply this measure to my newly designed ward which has not even been built, let alone used, yet?'. We developed SCI precisely to address this question. $\mathrm{SCl}$ incorporates the idea that accumulated connectivity is associated with communication instances, and helps generalise this information. As a result, the tool can also be used to test hospital ward layouts during design stages.

\section{Phase 2: developing SCl}

Following in the footsteps of the Yale Traffic Index and the model developed by Choudhary and her colleagues, ${ }^{23}$ we developed SCI by using our observational data to identify the most frequently traversed links between key areas in a hospital ward. We found four major links in total: (1) patient beds/nursing station, (2) patient beds/medicine room, (3) patient beds/patient 
beds, (4) nursing station/medicine room. These four links accounted for nearly $90 \%$ of all movement paths, regardless of ward size and type, staffing patterns, or bed capacities. These key areas would be found in every nursing unit. We therefore generalised our findings, assuming that the observation of other wards would produce comparable results.

We also worked with the reasonable assumption that nursing assignments would be clustered in space: The patients assigned to a single nurse would be located in close proximity to one another, and nurses would use the closest nursing station (if there was more than one). For example, if a ward had three nursing stations (Fig. 6), it would be effectively split into three assignments. Each of them would include: one of the nursing stations, the nearest medicine room (cabinet), and all patient rooms and bays in the closest proximity.

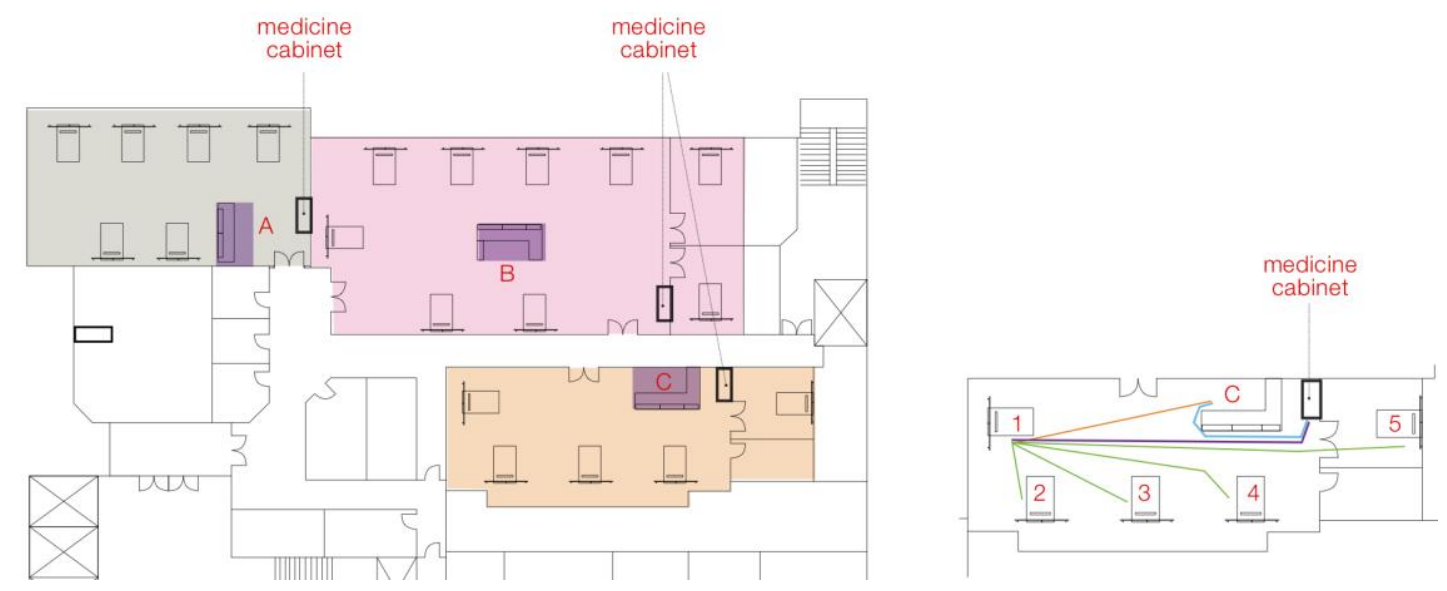

Figure 6. (left) Each assignment is marked in a different colour with nursing stations labelled as $\mathrm{A}, \mathrm{B}$ and $\mathrm{C}$, and all patient beds and the medicine cabinet that fall within the boundaries of the relevant colour; (right) the 'patient bed/nursing station' travel link for bed 1 is shown in orange, 'patient bed/ medicine cabinet' for the same bed number in purple, 'patient bed/ patient bed' in green and 'nursing station/medicine cabinet' in blue. The travel links for the other patient beds follow a similar principle

Only two critical variables were involved in calculating the index: the average connectivity of the paths that link key areas, and the times a path must be traversed. To measure the average connectivity of the path that connects the nursing stations to the medicine room, the connectivity of every pixel that falls within this path was recorded, and the average number was calculated. To calculate the average connectivity of a path between any two patient beds, we first connected each bed to every other bed, and then averaged the connectivity of those paths. We therefore assumed that visits to patient beds would be randomly distributed. In reality, the most serious cases are probably visited more often, but if we assume that patients are allocated to beds more or less randomly due to bed availability, the calculation seems reasonable. The same principle was applied to the links 'patient beds/nursing stations' and 'patient beds/medicine room'.

The relative number of times a path must be traversed can be represented by the percentage of total traffic it accounts for. We used this percentage as a factor (called 'a') to multiply the average connectivity levels 'aveConn' of each of the four major links. The path 'patient beds/patient beds' 
constituted $37 \%$ of total travel links in the detailed observation of the six NHS wards, 'patient beds/nursing station' constituted 29\%, 'patient beds/medicine room' accounted for $17 \%$ and 'nursing station/medicine room' accounted for $5 \%$. These percentages were used as frequency factors to multiply the average connectivity. For example, if the average connectivity of the path 'patient beds/ nursing station' was 230 , this number was then multiplied by 29 . The resulting values represented the possible accumulated connectivity along any of the four paths during a 24-hour shift. They could be used as a proxy for number of conversations. The four values were then summed up. To account for the ward unit size, we divided them by the number of patient beds. The result comprises the Spaces for Communication Index. This was done for every nursing assignment. So, if there were more than one assignments in a ward, we summed the four values for all assignments, and then divided the total by the number of patient beds.

$$
\begin{aligned}
& S C I=\frac{\sum_{i=1}^{4} \text { aveConn }_{i} a_{i}}{N} \quad \begin{array}{l}
\text { where aveConn } \\
\text { is the average connectivity of the four links }
\end{array} \\
& a_{i} \text { is the frequency factor for the relevant link } \\
& N \text { is the number of beds in the ward }
\end{aligned}
$$

\section{Phase 3: testing SCI on larger sample}

Our hypothesis was that the unit with the best quality of care would be the one on which care providers accumulated the maximum connectivity levels in any 24-hour period. To test it, we calculated the index for a larger sample of thirty-one NHS case studies. The resulting figures were then related to the quality of care provided by the hospital (Fig. 7). We also tested if the number of patient beds and the size of the ward had any effect on care quality.

Finally, we used the same thirty-one case studies to calculate the Yale Traffic Index that also accounted for travel distances between functional areas, ${ }^{24}$ and tested for their potential effect on care quality. Ordinal regression analysis, the most appropriate statistical model for our dataset, was used to investigate the relationship. Results showed that $\mathrm{SCI}$ was fairly successful in establishing which wards provided better healthcare quality $(p=0.005)$. It can therefore be used as a tool to assess layouts, and anticipate certain levels of care quality. The higher the index, the better the quality of care. Ward size and number of patient beds were not indicators of healthcare quality, on their own ( $p$ $=0.304$ and $p=0.237$, respectively) ${ }^{25}$ These results are in line with findings from previous studies, where ward size or number of beds did not yield significant results. ${ }^{26}$ Likewise, the Yale Traffic Index did not have any effect on healthcare quality in this sample $(p=0.693)$. 

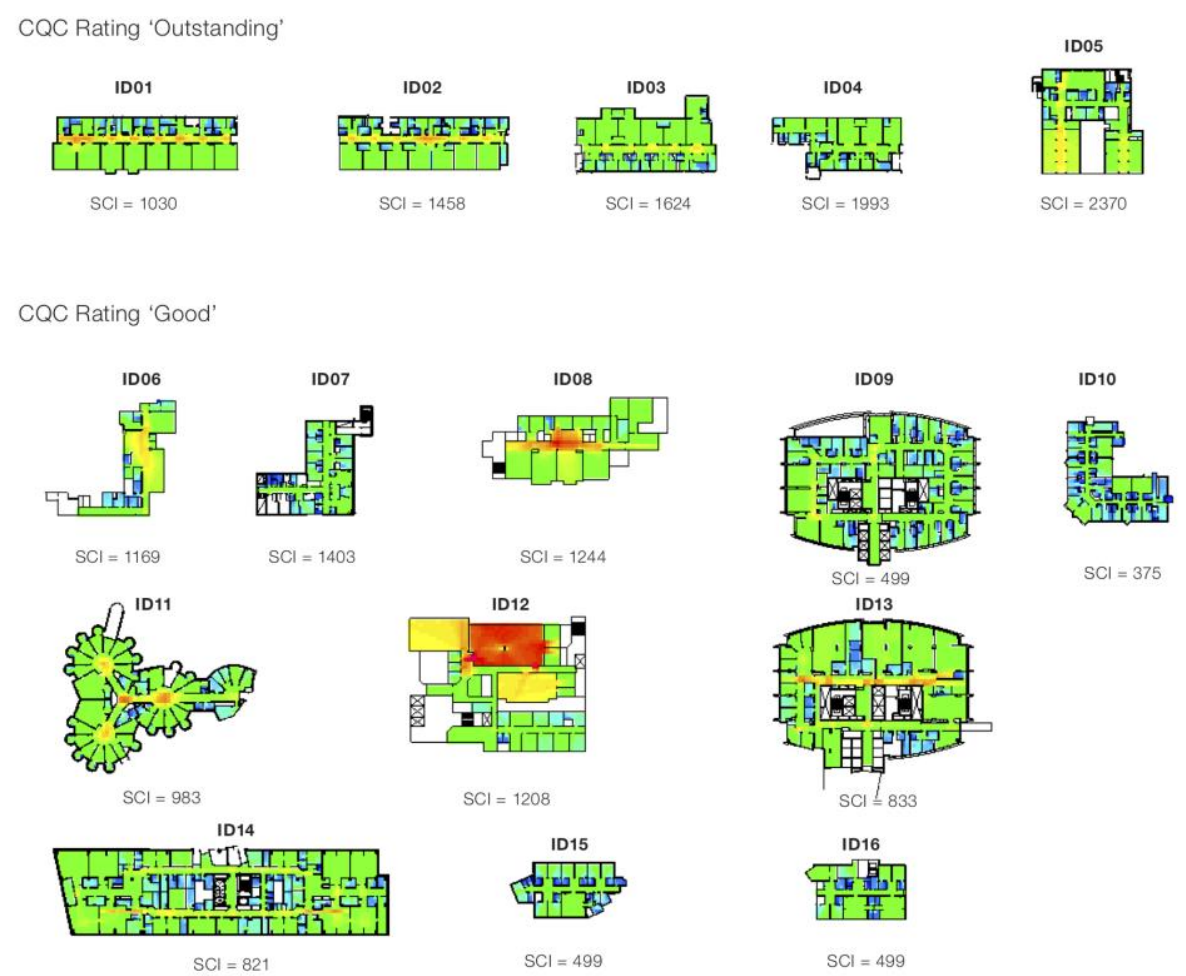

CQC Rating 'Requires Improvement' + 'Inadequate'

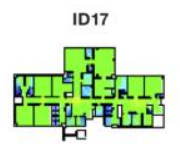

$\mathrm{SCl}=615$

ID22

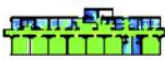

$\mathrm{SCl}=847$

ID27

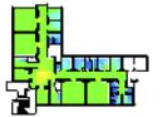

$\mathrm{SCl}=1201$

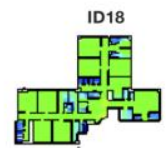

$\mathrm{SCl}=637$

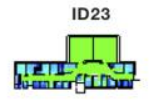

$\mathrm{SCl}=887$

ID28

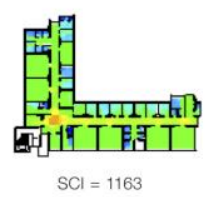

ID19

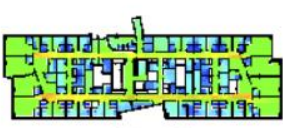

$\mathrm{SCl}=432$

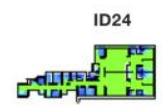

$\mathrm{SCl}=906$

ID29

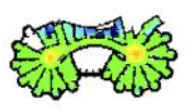

$\mathrm{SCl}=1071$
ID20

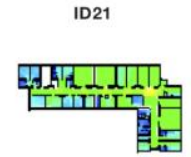

$\mathrm{SCl}=793$

ID26

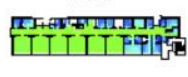

$\mathrm{SCl}=974$

ID30

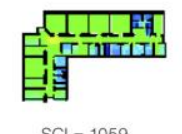

$\mathrm{SCl}=1059$

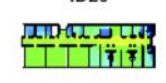

$\mathrm{SCl}=1404$

ID31

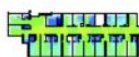

$\mathrm{SCl}=1252$

Figure 7. Thirty-one NHS hospital wards used for testing $\mathrm{SCl}$ ordered by quality of care rating as given by the Care Quality Commission (CQC). The SCl value is provided for each hospital ward. The connectivity of each layout is illustrated where warm colours show more connected areas and cooler colours indicate areas with fewer connections. All wards are on the same colour scale

\section{Conclusions: the significance of $\mathrm{SCl}$}

In terms of design, these results suggest that ward size is not a predictor of quality of care. More important is the openness of spaces that healthcare workers traverse to get from one key area to another. The larger in size the area that links one key function to another, and the larger the viewsheds it 
provides, the higher the chances of bumping into another healthcare worker, and having an informal, quick conversation about important patient information. Wards with a higher SCI value, i.e. with larger viewsheds on key paths, were more likely to be rated as 'outstanding' or 'good' than wards with a lower SCI value. Thus, the spatial layouts of hospital wards were shown to be associated with quality of care ratings. The findings of this research also make sense intuitively. Spaces with larger viewsheds allow for frequent and spontaneous exchanges among healthcare workers. Even just social chitchat, exchanging smiles, or saying 'hi' can contribute to a productive workplace environment. By contrast, efficiency-based measures such as distances between functional areas seemed to have a less direct influence on healthcare quality.

This research contributed to the development of an objective method that can be used for benchmarking design options. These could help any decisionmaker involved in building a hospital. Decisions between different layouts should be driven by how well these might support provision of good healthcare. The newly developed SCI index allows for a systematic comparison of hospital ward layouts during the design stage. Designers and hospital planners could use this to inform their designs, and make choices that maximise communication opportunities, which in turn render better care quality more likely. Rather than considering construction cost or walking efficiencies, this new tool presents healthcare planners and designers with a different perspective. $\mathrm{SCl}$ may hold an answer to the main questions: 'Why are there so many different layouts? Which one works best?', as it offers an outcome- and qualitydriven view. Thirty-one plans may be a small sample to draw certain conclusions from. More case studies would be required for testing and finetuning the measure. But the existing sample is still a great start, and forms a basis for further application.

Next steps for this research project include applying the index calculation during the design and planning stages of hospitals to optimise for communication opportunities. This should help designers know in advance how much they must invest in spatial configuration to provide better healthcare quality by design.

\section{Acknowledgements}

Special thanks to Petros Koutsolampros for developing an R script to automate the process of calculating distances and connectivity values between key areas in a hospital ward. Without this software, it would not have been possible to apply the new measure on a larger sample.

\section{Notes and references}

1. Ann Hendrich, Marilyn P. Chow, Boguslaw A. Skierczynski and Zhenqiang Lu, 'A 36-Hospital Time and Motion Study: How Do Medical-Surgical Nurses Spend Their Time?', The Permanente Journal, 12(3) (2008), 25-34.

2. Kirk D. Hamilton and Mardelle M. Shepley, Design for Critical Care: An Evidence-Based Approach (Oxford: Architectural Press, 2010).

3. Yoel Donchin, Daniel Gopher, Miriam Olin, Yehuda Badihi, MR Biesky, Charles Sprung, Reuven Pizov and Shamay Cotev, 'A Look into the Nature and Causes 
of Human Errors in the Intensive Care Unit', Quality \& Safety in Health Care, 12 (2003), 143-47.

4. William A. Knaus, Elizabeth A. Draper, Douglas P. Wanger and Jack E. Zimmerman, 'An Evaluation of Outcome from Intensive Care in Major Medical Centers', Annals of Internal Medicine, 104(3) (1986), 410-18.

5. Madelyne Sturdavant, Comparisons of Intensive Nursing Service in a Circular and a Rectangular Unit, Rochester Methodist Hospital, Rochester, Minn (Chicago: American Hospital Association, 1960).

6. David Trites, Frank Galbraith, John Leckwart and Medelyne Sturdavant, 'Radial Nursing Units Prove Best in Controlled Study', Modern Hospital, 112 (1969), 94-99; David Trites, Frank Galbraith, Medelyne Sturdavant and John Leckwart, 'Influence of Nursing-unit Design on the Activities and Subjective Feelings of Nursing Personnel', Environment and Behavior, 2 (1970), 303-34.

7. John Freeman, 'Quantitative Criteria for Hospital Inpatient Nursing Unit Design' (unpublished doctoral thesis, Georgia Institute of Technology, 1967).

8. Richard Sendler, 'Quantitative Evaluation of Inpatient Nursing Unit Designs' (unpublished doctoral thesis, Georgia Institute of Technology, 1968).

9. John D. Thompson and Grace Goldin, The Hospital: A Social and Architectural History (New Haven, CT: Yale University Press, 1975).

10. See Rosamund Cama, Evidence-Based Healthcare Design (NJ: Wiley, 2009); Hamilton and Shepley, Design for Critical Care.

11. Bill Hillier and Julienne Hanson, The Social Logic of Space (Cambridge: Cambridge University Press, 1984).

12. See Alan Penn, Justin Desyllas and Laura Vaughan, 'The Space of Innovation: Interaction and Communication in the Work Environment', Environment and Planning B: Planning and Design, 26(2)(1999), 193-218; Kerstin Sailer and Alan Penn, 'Spatiality and Transpatiality in Workplace Environments', in Proceedings of the 7th International Space Syntax Symposium, ed. by Daniel Koch, Lars Marcus and Jesper Steen (Stockholm: KTH, 2009); Mahbub Rashid, Kevin Kampschroer, Jean Wineman and Craig Zimring, 'Spatial Layout and Face-to-Face Interaction in Offices: A Study of the Mechanisms of Spatial Effects on Face-to-Face Interaction', Environment and Planning B: Planning and Design, 33 (2006), 825-44.

13. Ruchi Choudhary, Sonit Bafna,Yeonsook Heo, Ann Hendrich and Marilyn Chow,'A Predictive Model for Computing the Influence of Space Layouts on Nurses' Movement in Hospital Units', Journal of Building Performance Simulation, 3(3) (2010), 171-84.

14. Yi Lu, John Peponis and Craig Zimring, 'Targeted Visibility Analysis in Buildings: Correlating Targeted Visibility Analysis with Distribution of People and Their Interactions within an Intensive Care Unit', in Proceedings of the 7th International Space Syntax Symposium, ed. by Daniel Koch, Lars Marcus and Jesper Steen (Stockholm: KTH, 2009), 68.1-68.10.

15. See Yi Lu, Michelle M. Ossmann, David E. Leaf and Philip H. Factor, 'Patient Visibility and ICU Mortality: A Conceptual Replication', Health Environments Research \& Design Journal, 7(2) (2014), 92-103; David E. Leaf, Peter Homel and Phillip Factor, 'Relationship between ICU Design and Mortality', CHEST Journal, 137(5) (2010), 1022-27.

16. Mahbub Rashid, Diane Boyle and Michael Crosser, 'Network of Spaces and Interaction- Related Behaviors in Adult Intensive Care Units', Behavioral sciences, 4 (2014), 487-510.

17. Hillier and Hanson, The Social Logic.

18. Lu, Peponis and Zimring, 'Targeted Visibility Analysis'; Rashid, Boyle and Crosser, 'Network of Spaces'.

19. Michael L. Benedikt, 'To Take Hold of Space: Isovists and Isovist Fields', Environment and Planning B, 6(47) (1979), 47-65. 
20. depthmapX development team, depthmapX (Version 0.6.0) (2017), Space Group UCL.

21. Alasdair Turner, Maria Doxa, David O'Sullivan and Alan Penn, 'From Isovists to Visibility Graphs: A Methodology for the Analysis of Architectural Space', Environment and Planning

22. Lu, Peponis and Zimring, 'Targeted Visibility Analysis'; Yi Lu and Craig Zimring, 'Can Intensive Care Staff See Their Patients? An Improved Visibility Analysis Methodology', Environment and Behavior, 44(6) (2012), 861-76.

23. Choudhary, Bafna, Heo, Hendrich and Chow, 'A Predictive Model'.

24. Thompson and Goldin, The Hospital, p. 290.

25. In statistics, only results with $p$-value $<0.05$ are considered significant, i.e. when there is lessispithan $5 \%$ chance that a random variable could explain the result.

26. Thompson and Goldin, The Hospital; Hyun-Bo Seo, Young-Seon Choi and Craig Zimring, 'Impact of Hospital Unit Design for Patient-Centered Care on Nurses' Behavior', Environment and Behavior, 43(4) (2011), 443-68. 